\title{
Portal Vein Occlusion Prior to Extensive Resection in Colorectal Liver Metastasis: A Necessity Rather than an Option!
}

\author{
Jacques Belghiti, $\mathrm{MD}^{1,2}$ and Léonor Benhaïm, $\mathrm{MD}^{1,2}$ \\ ${ }^{1}$ Department of HBP Surgery, Hospital Beaujon, Clichy, France; ${ }^{2}$ University Paris 7 Denis Diderot, Paris, France
}

Preoperative portal vein occlusion (PVO) of one liver lobe is regularly used for inducing liver hypertrophy of the contralateral lobe to prevent postoperative liver failure in patients with an anticipated insufficient future liver remnant (FLR) following resection. ${ }^{1} \mathrm{~A}$ similar rate of hypertrophy of the liver parenchyma with preserved portal flow can be obtained either by percutaneous portal embolization using several agents or by portal vein ligation. ${ }^{2}$ This strategy of preoperatively manipulating the liver volume has been acclaimed as a major step in liver surgery, paving the way for extensive resection for initially unresectable tumors. ${ }^{3}$

It has been clearly shown that chemotherapy of colorectal liver metastases (CRLM) can provide significant downstaging of liver disease enabling curative rescue resection and translating into an improved long-term survival. ${ }^{3}$ Over the last decade, both PVO and chemotherapy have dramatically widened the scope of surgical resection of CRLM. Several institutions involved in the surgical treatment of CRLM have claimed that preoperative PVO has increased the number of candidates for complete tumor resection. ${ }^{3-5}$ The hypertrophy of the FLR allows the safety of major resection.

In the present issue, the article by Pamecha et al. tends to dampen the early enthusiasm regarding PVO for CLRM. ${ }^{6}$ This well-written article, which compares the surgical outcomes of two groups of patients operated for CRLM with or without portal vein embolization (PVE), highlights the unfavorable effects of PVE. Firstly, one-third of the patients were nonoperable after PVE because of rapid progression of disease, and secondly the long-term

(C) Society of Surgical Oncology 2009

Published Online: 25 February 2009

J. Belghiti, MD

e-mail: jacques.belghiti@bjn.aphp.fr survival for patients resected after PVE was less favorable than for the group who underwent immediate surgery.

A legitimate concern is whether the stimulus for liver regeneration induced by PVO might enhance tumor growth. As reported in a recent review of this journal, experimental studies have shown that hepatectomy or portal ligation in rats induced tumor growth and in some clinical studies, after PVO, the growth rate of liver metastasis seems to be more rapid than that of the liver parenchyma including a higher proliferative activity of intrahepatic metastasis possibly induced by hyper arterializations. ${ }^{7}$

Although these studies clearly demonstrate that tumor progression after PVO is possible, we believe that it is not yet time to consider a restriction of preoperative modulation of the liver parenchyma by PVO. Percutaneous embolization is a safe procedure that could reduce intrahepatic recurrence rate after right hepatectomy for unilobar CRLM. ${ }^{8}$ Several series have shown that in patients with extensive CRLM who would otherwise be unresectable, PVO followed by surgery offers a significant benefit with a 5-year survival up to $40 \% .^{5,8-11}$ In these studies the evidence of direct stimulation of tumor growth by PVE is circumstantial, and the rate of unresectability after PVE due to intrahepatic and/or extrahepatic tumor progression is regularly around $25 \%$, which is not very different from the results of Pamecha et al. ${ }^{6}$

The main problem raised by this article is the indication of PVO and its association with chemotherapy before a major liver resection. The indication of PVO should depend on several factors including the extent and expected difficulties of resection, the status of nontumorous liver parenchyma, and the exact quantification of sufficient minimal functional hepatic volume. We have clearly demonstrated that a right hepatectomy, which is the main procedure performed after PVO, can be safely performed in patients with normal parenchyma. ${ }^{12}$ Therefore, except in patients with a FLR less than $25 \%$ of the total volume and 
those requiring an associated procedure in the FLR, there is no indication of preoperative PVO. The same prospective study has shown that PVO is beneficial in patients with a diseased parenchyma. As a matter of fact, nowadays, nearly all patients operated for CRLM had received preoperative chemotherapy that could induce parenchymal changes altering the regenerative capacity of the FLR. Although the impact of these various changes on the outcome of the liver function remains debatable, there are some arguments to consider PVO in patients who received numerous cycles of chemotherapy. ${ }^{13}$ This approach, which requires further study, could be established according to results of preoperative biopsy of the nontumorous liver.

We agree with Pamecha et al. that in patients with bilobar CRLM considered for a right hepatectomy, the presence of tumors in the left liver requires specific consideration. Firstly, chemotherapy after PVE should not be interrupted. There are some data suggesting that chemotherapy does not impair hypertrophy of the left liver after right $\mathrm{PVO}$ and may be continued after completion of the PVO. ${ }^{14}$ Another possibility is to ablate small CRLM in the FLR by percutaneous radiofrequency before PVO. In cases of large CRLM in the FLR, a two-stage surgical procedure can be recommended. ${ }^{9}$ In the first stage, all visible metastases in the left hemiliver are cleared in association with right portal ligation, and in the second stage a right or extended right hemihepatectomy is performed after use of concomitant chemotherapy, excluding bevacizumab.

In summary, the benefits of a potential cure after a complete surgical resection after PVO in extensive CRLM outweigh the risks of tumor progression since these patients are otherwise unresectable. PVO should be strictly limited to those with a significant risk of postoperative liver insufficiency. Hence, rather than an optional procedure, it is a compulsion in this group of patients to facilitate a possible curative resection. Encouraging long-term survival after complete resections in patients who are "unresectable," coupled with the ever-expanding horizons of chemotherapy, clearly tilt the balance in favor of preoperative liver volume modulation. Future studies should focus on strategies to reduce the risk of disease progression after PVO. Tumor progression during chemotherapy is clearly a negative prognostic factor that should help filter the likely beneficiaries. A step further in patient selection may be to ascertain the rate of tumor necrosis induced by chemotherapy. ${ }^{15}$ These strategies may enable a more selective approach in offering PVO and subsequent surgery to those who are more likely to benefit from it.

\section{REFERENCES}

1. Abulkhir A, Limongelli P, Healey AJ, Damrah O, Tait P, Jackson $\mathrm{J}$, et al. Preoperative portal vein embolization for major liver resection: a meta-analysis. Ann Surg. 2008;247:49-57.

2. Aussilhou B, Lesurtel M, Sauvanet A, Farges O, Dokmak S, Goasguen N, et al. Right portal vein ligation is as efficient as portal vein embolization to induce hypertrophy of the left liver remnant. J Gastrointest Surg. 2008;12:297-303.

3. Azoulay D, Castaing D, Smail A, Adam R, Cailliez V, Laurent A, et al. Resection of nonresectable liver metastases from colorectal cancer after percutaneous portal vein embolization. Ann Surg. 2000;231:480-6.

4. Kawasaki S, Makuuchi M, Kakazu T, Miyagawa S, Takayama T, Kosuge $\mathrm{T}$, et al. Resection for multiple metastatic liver tumors after portal embolization. Surgery. 1994;115:374-7.

5. Jaeck D, Oussoultzoglou E, Rosso E, Greget M, Weber JC, Bachellier P. A two-stage hepatectomy procedure combined with portal vein embolization to achieve curative resection for initially unresectable multiple and bilobar colorectal liver metastases. Ann Surg. 2004;240:1037-49.

6. Pamecha V, Glantzounis G, Davies N, Fusai G, Sharma D, Davidson B. Long term survival and disease recurrence following portal vein embolisation prior to major hepatectomy for colorectal metastases. Ann Surg Oncol. 2009; Jan 6 (Epub ahead of print).

7. de Graaf W, van den Esschert JW, van Lienden KP, van Gulik TM. Induction of tumor growth after preoperative portal vein embolization: is it a real problem? Ann Surg Oncol. 2009;16:42330.

8. Oussoultzoglou E, Bachellier P, Rosso E, Scurtu R, Lucescu I, Greget $\mathrm{M}$, et al. Right portal vein embolization before right hepatectomy for unilobar colorectal liver metastases reduces the intrahepatic recurrence rate. Ann Surg. 2006;244:71-9.

9. Adam R, Laurent A, Azoulay D, Castaing D, Bismuth H. Twostage hepatectomy: a planned strategy to treat irresectable liver tumors. Ann Surg. 2000;232:777-85.

10. Mueller L, Hillert C, Möller L, Krupski-Berdien G, Rogiers X, Broering DC. Major hepatectomy for colorectal metastases: is preoperative portal occlusion an oncological risk factor? Ann Surg Oncol. 2008;15:1908-17.

11. Elias D, Ouellet JF, De Baère T, Lasser P, Roche A. Preoperative selective portal vein embolization before hepatectomy for liver metastases: long-term results and impact on survival. Surgery. 2002;131:294-9.

12. Farges O, Belghiti J, Kianmanesh R, Regimbeau JM, Santoro R, Vilgrain V, et al. Portal vein embolization before right hepatectomy: prospective clinical trial. Ann Surg. 2003;23:208-17.

13. Clavien PA, Petrowsky H, DeOliveira ML, Graf R. Strategies for safer liver surgery and partial liver transplantation. $N$ Engl J Med. 2007;356:1545-59.

14. Goéré D, Farges O, Leporrier J, Sauvanet A, Vilgrain V, Belghiti J. Chemotherapy does not impair hypertrophy of the left liver after right portal vein obstruction. $J$ Gastrointest Surg. 2006; 10:365-70.

15. Blazer DG 3rd, Kishi Y, Maru DM, Kopetz S, Chun YS, Overman MJ, et al. Pathologic response to preoperative chemotherapy: a new outcome end point after resection of hepatic colorectal metastases. J Clin Oncol. 2008;26:5344-51. 